\title{
Waveguide insulator - theory and experiment
}

\author{
Ruslana Zalutskaya ${ }^{1^{*}}$, and Mikhail Mironov ${ }^{1,2}$ \\ ${ }^{1}$ Bauman Moscow State Technical University, 105005, MoscowRussia \\ ${ }^{2}$ Andreyev Acoustics Institute, 117036, Moscow, Russia
}

\begin{abstract}
The theory of sound propagation in waveguides with a system of resonators on the wall is given in [1-2]. The papers present theoretical data showing that the use of a chain of identical Helmholtz resonators on the walls of a waveguide reduces the sound pressure levels effectively not only at the resonant frequency, but also at frequencies which are significantly higher than the resonant frequency. This theory was called by the authors of $[1,2]$ the theory of waveguide isolation (WI). N owadays, the decades later, the idea of using a set of identical resonators has been widely developed in creation and research of acoustic metamaterials - artificial structures with unusual wave properties (see for example [3-6]). A t the same time, the works [1-2] remained al most unnoticed. This paper is devoted to experimental and numerical verification of the WI theory. The design of the studied WI, a simple experimental setup, the results of the experiment and numerical experiment are described.
\end{abstract}

\section{The experimental waveguide insulator and experimental plant}

The investigated model of a waveguide insulator (WI) is a small-diameter tube with three identical Helmholtz resonators attached to the pipe wall. The chambers of each resonator are connected with the tube by means of six holes in the tube wall. The design of the WI with the main dimensions is shown in Figure 1.

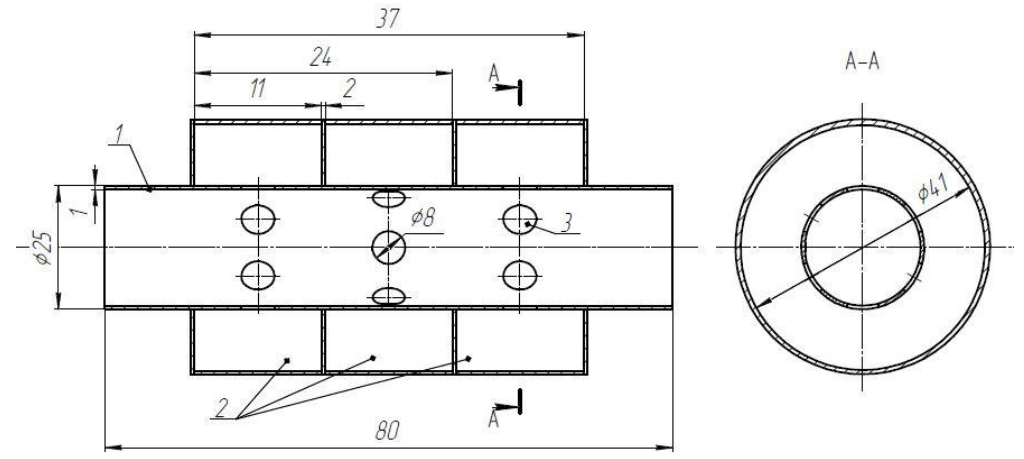

Fig. 1. The design of the waveguide insulator.

* Corresponding author: author@email.org 
To reduce the background noise in the laboratory, the box with the inner walls lined with sound absorbing material is used. The scheme of the experimental installation is shown in Figure 2 .

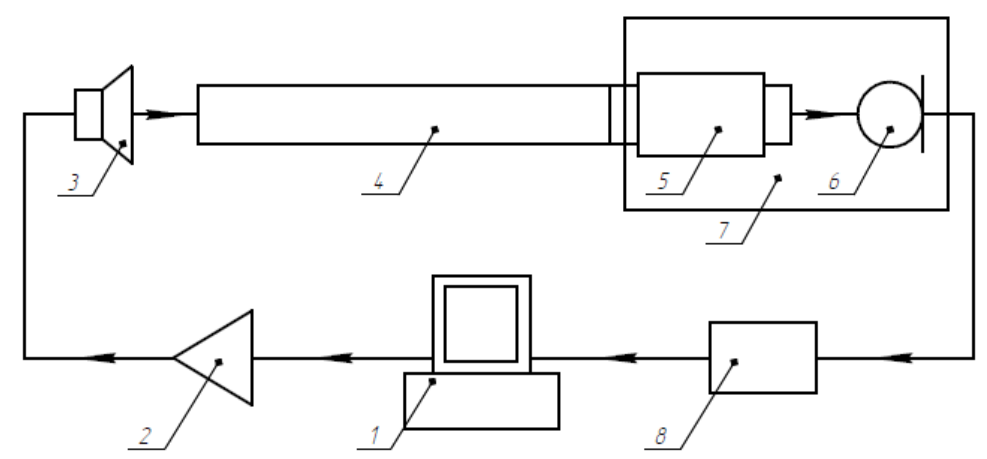

Fig. 2. Scheme of the experimental installation.

The operational principle of the setup is the following: a signal with a linear frequency modulation (LFM) from the computer (1), after passing through the amplifier (2), is fed to a speaker (3). The sound generated by the speaker propagates through the pipe 4 , then passing through a WI (5), is recorded by the microphone (6). The outlet of the WI and a microphone are placed in a special box (7) coated with a layer of sound-absorbing material. The signal from the microphone is sent via the sound card (8) to the computer, where the signal is recorded with the SpectraLab program. Then the program constructs the signal spectrum with Fourier transform. The generated frequency band of the LFM signal is $1 \mathrm{kHz}-8 \mathrm{kHz}$.

A $0.8 \mathrm{~m}$ long tube was used between the speaker, and the waveguide isolator (Figure 3).

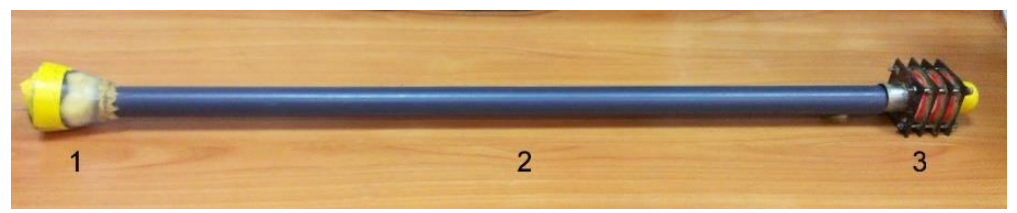

Fig. 3. Model of the WI 3 in the assembly with the speaker 1 and the tube 2.

\section{Experimental results}

The measured spectra of the transmitted sound at the same voltage applied to the speaker are shown in Figure 4. Top line 1 corresponds to the levels of the sound passed through the tube, the middle line 2 - to the levels of sound transmitted through a tube with attached WI, the lower one 3 - to the background noise. The characteristic amplitude fluctuations on the graphs 1 and 2 are associated with the tube resonances. The graphs shows that the sound level is comparable to the background noise levels at the frequencies close to the natural resonant frequency of the isolator $f \sim 3600 \mathrm{~Hz}$ (see below the formula (1)). That is, the insulator "locks" the noise emitted by the speaker almost completely. 


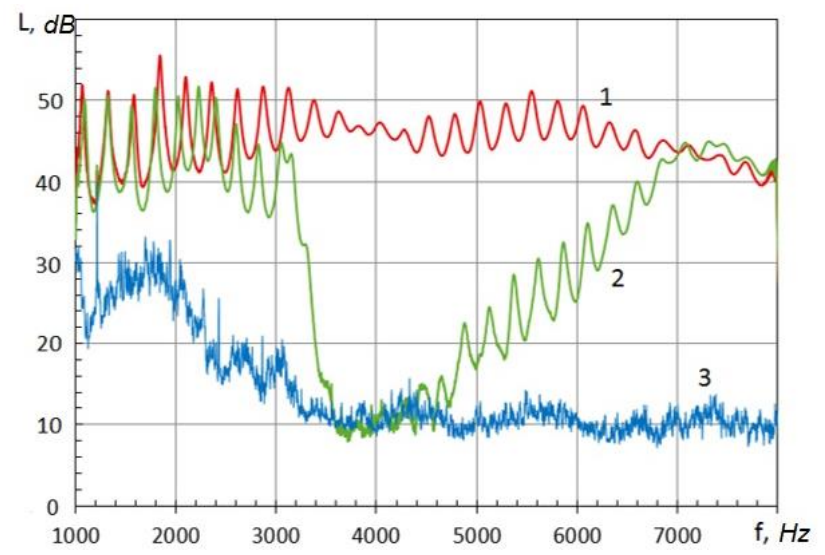

Fig. 4. The spectra of the passed noise: 1 - tube; 2 - tube with WI; 3 - background noise.

The efficiency of a waveguide insulator (Figure 5) is equal to the difference between the sound levels passed through the tube (curve 1) and the sound levels passed through the tube with a WI (curve 2).

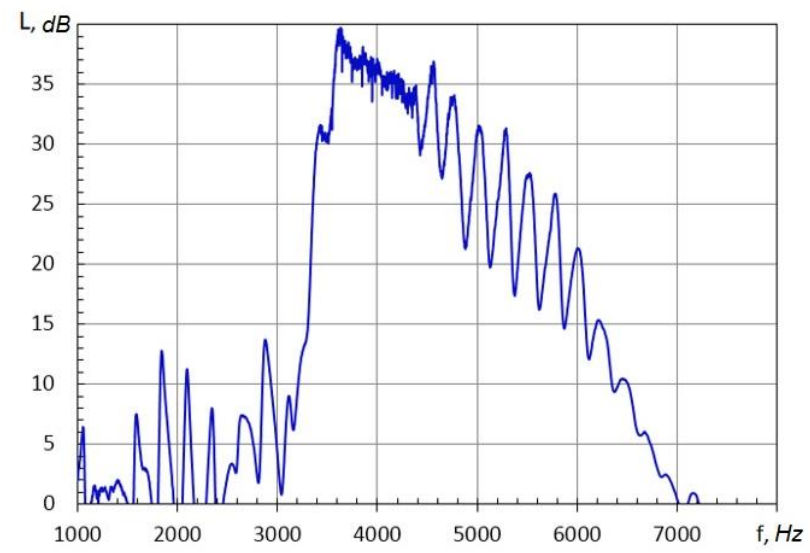

Fig. 5. The efficiency of a WI.

The maximum sound reduction occurs at frequencies close to the eigen frequency of the system, and is equal to $35-39 \mathrm{~dB}$ approximately. In addition, the action of the WI continues at higher frequencies and ends at frequencies of 6.6-7 kHz.

\section{Numerical modeling}

The numerical simulation of a WI was performed in the COMSOL Multiphysics software package, which is widely used for acoustic calculations [7-10]. The program is based on the finite element method. The whole volume under investigation is divided into a set of elementary volumes and the sound pressure is calculated in each elementary volume.

At the first stage of modeling, the geometry of a WI model was constructed taking into account all the dimensions of the structure, given in Figure 1. Then the values of all the necessary parameters were entered and the boundary conditions at the input and output of the WI were set. The finite element model of a WI is shown in Figure 6. 


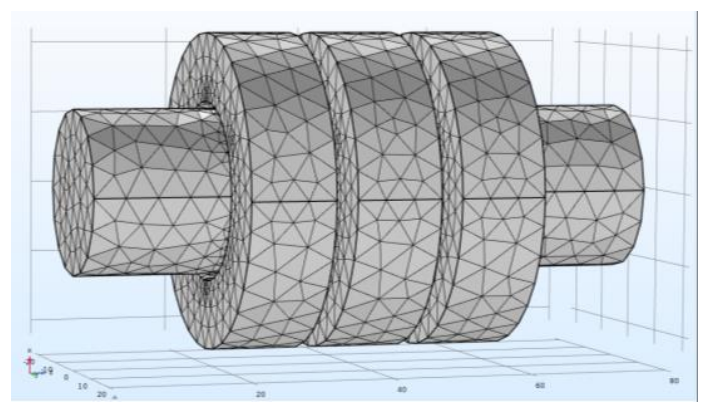

Fig. 6. The finite element model of a WI.

The efficiency of the WI is determined as the sound energy transmission loss (TL), which is ten logarithms of the ratio of the sound power of the direct wave entering the insulator input to the sound power of the direct wave at the output of the insulator. The graph of the calculated WI transmission losses is shown in Figure 7.

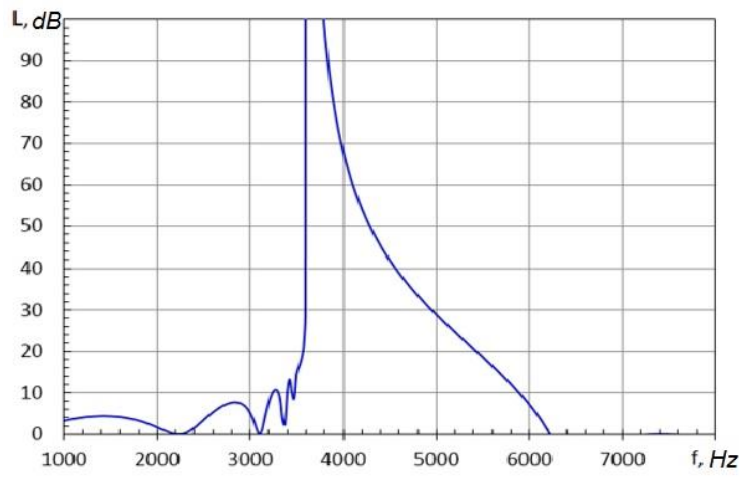

Fig. 7. WI transmission losses.

The examples of spatial distribution of sound pressure levels in a WI for different frequencies are shown in fig. 8.1-8.3.

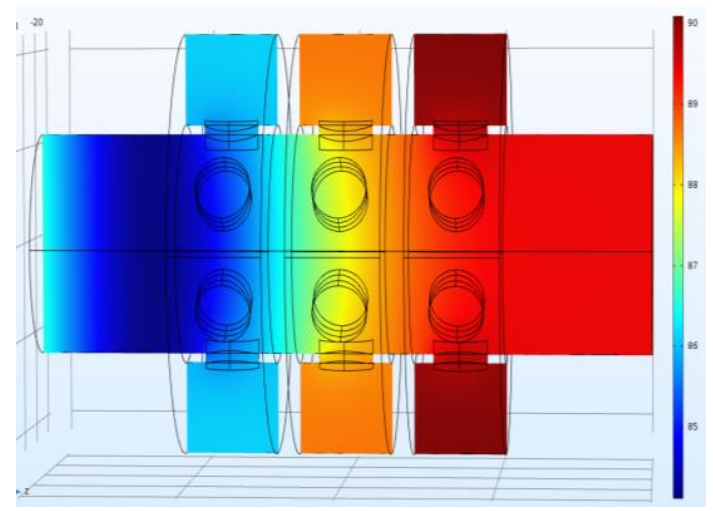

Fig. 8. The SPL distribution inside the waveguide isolator for $1 \mathrm{kHz}$. 


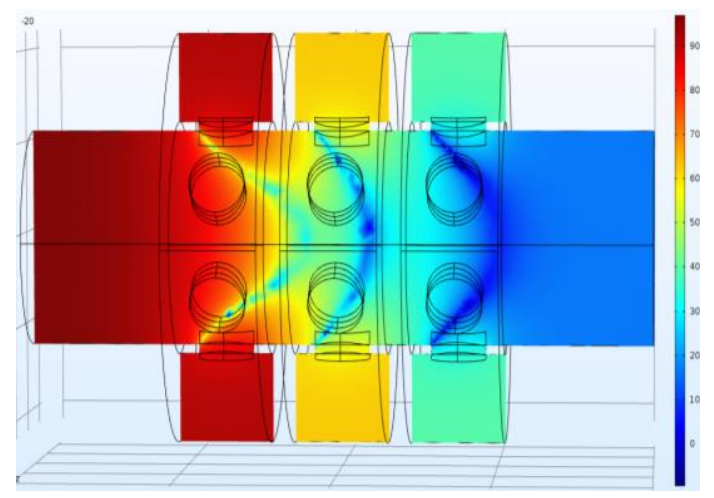

Fig. 8. The SPL distribution inside the waveguide isolator for $3,6 \mathrm{kHz}$.

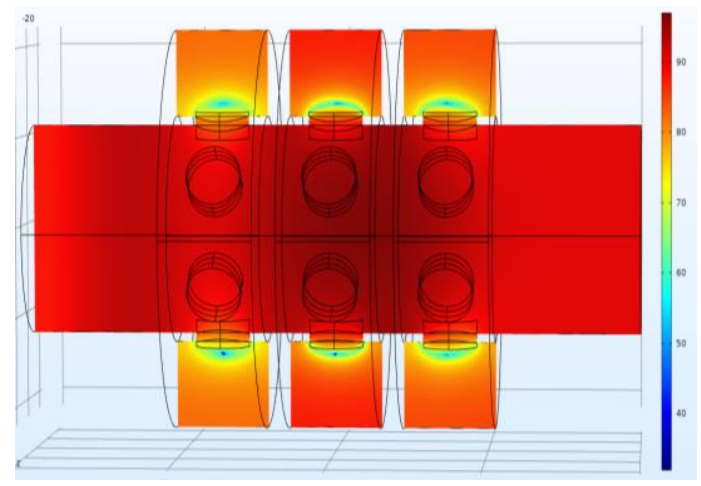

Fig. 9. The SPL distribution inside the waveguide isolator for $7 \mathrm{kHz}$.

The first picture (Figure 8) corresponds to a wave with a frequency of $1 \mathrm{kHz}-3.5$ times lower than the resonant frequency. The wave passes through the isolator without changes (the full color scale in this figure is $10 \mathrm{~dB}$ ).

The second picture (Figure 9) corresponds to a wave with a frequency equal to the eigen frequency of the WI. At this frequency, the insulator has the highest efficiency and, according to the picture, the sound pressure level at the output is reduced by an amount approximately equal to $70 \mathrm{~dB}$ (full scale $-95 \mathrm{~dB}$ ).

The third picture (Figure 10) corresponds to a wave with a frequency of $7 \mathrm{kHz}$. WI is ineffective at this frequency (the full scale is $40 \mathrm{~dB}$ ).

\section{Analytical results}

Calculations of WI were also performed within the one-dimensional analytical model suggested in $[1,2]$.

The resonant frequency of one resonator in the considered model is equal to:

$$
f_{0}=\frac{1}{2 \pi} \sqrt{\frac{S}{L \cdot V}} \approx 3.6 \mathrm{kHz}
$$

where $S$ - the area of all resonator holes, the diameter of each hole is $8 \mathrm{~mm}$, the wall thickness of the tube is $1 \mathrm{~mm} ; L$ - the effective neck length of the resonator, which is the sum of the neck length and the attached neck length: $L \approx 7.2 \mathrm{~mm} ; V=9.1 \cdot 10^{-6} \mathrm{~m}^{3}$ - resonator volume.

The field in the tube is presented as the sum of the incident and reflected waves: 


$$
\begin{aligned}
& p_{1}=A_{1} \exp (i k x)+B_{1} \exp (-i k x) \\
& u_{1}=\frac{1}{\rho c}\left[A_{1} \exp (i k x)-B_{1} \exp (-i k x)\right]
\end{aligned}
$$

The field in the WI is:

$$
\begin{aligned}
& p_{2}=A_{2} \exp (i \xi x)+B_{2} \exp (-i \xi x) \\
& u_{2}=\frac{\xi}{\rho \omega}\left[A_{2} \exp (i \xi x)-B_{2} \exp (-i \xi x)\right]
\end{aligned}
$$

According to the [1], the longitudinal wave number $\xi$ in (4) and (5) is determined by the formula:

$$
\left(\frac{\xi}{k_{0}}\right)^{2}=\left(\frac{\omega}{\omega_{0}}\right)^{2} \frac{N^{2}-\left(\omega / \omega_{0}\right)^{2}}{\left(\omega / \omega_{0}\right)^{2}-1}
$$

where $k_{0}=\frac{\omega_{0}}{c} ; \omega_{0}-$ eigen frequency of resonator;

$$
N=\sqrt{\frac{\Omega}{\pi a^{2}}+1}
$$

where $\Omega=\pi \cdot\left(b^{2}-a^{2}\right)$ the volume of the resonator chamber per unit length of the tube, $a-$ tube radius, $b$ - radius of the external resonator shell.

The parameter $N$ included in (6) determines the width of the waveguide locking frequency band. Indeed, when the right side of equation (6) is negative, the wave number is imaginary and the wave does not propagate in the frequency range $\omega_{0}<\omega<N \omega_{0}$. The wave amplitude decreases exponentially along the waveguide axis as $\exp (-|\xi| \cdot x)$.

For these parameters of the WI $N=1.73$. In this case, the lower boundary of the locking band according to (1) is equal to $f_{l}=3.6 \mathrm{kHz}$, and its upper boundary is equal to $f_{h}=6.2 \mathrm{kHz}$.

The dependence of the WI acoustic efficiency on the frequency may be calculated analytically. Writing down the boundary conditions and solving the corresponding system of equations, an expression for calculating the oscillation velocity at the WI exit can be obtained (because of its bulkiness, it is not given here).

Using the obtained expression, the calculated efficiency of a waveguide isolator can be found. It is equal to twenty logarithms of the ratio of the vibrational velocity at the tube outlet to the vibrational velocity at the WI outlet (Figure 9).

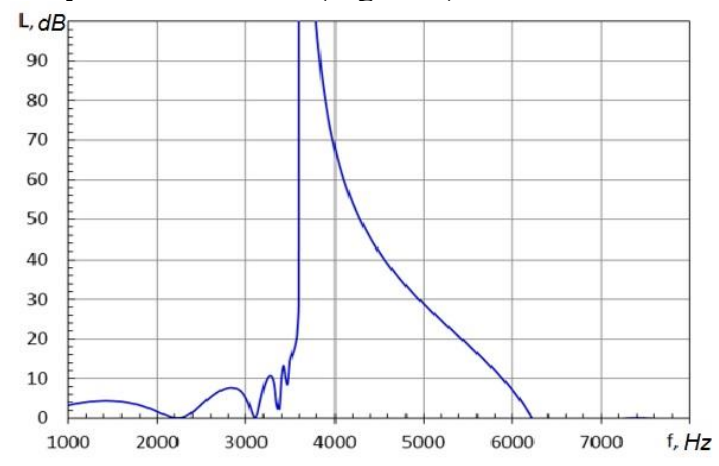

Fig. 11. Theoretical efficiency of a WI.

The analysis shows that the graphs of the waveguide isolator efficiency obtained experimentally (Figure 5), by finite element modeling (Figure 7) and as a result of analytical calculations (Figure 9) are comparable with each other. The operating range of the WI is 
observed at frequencies from $3000 \mathrm{kHz}$ to $6.5 \mathrm{kHz}$. The maximum sound insulation is observed at the WI eigen frequency, which is equal to $3.6 \mathrm{kHz}$, and actually reaches values of 35-39 dB. The WI efficiency obtained experimentally has a slightly larger frequency width than in Figure 7 and Figure 9.

\section{Conclusion}

1. The investigated WI is effective in a wide frequency range. The maximum efficiency is observed at frequencies close to the resonant frequency and is $35-40 \mathrm{~dB}$. The actual locking frequency range of the WI (more than $15 \mathrm{~dB}$ sound reduction) is observed at the frequency band from $3.3 \mathrm{kHz}$ to $6.2 \mathrm{kHz}$.

2. The results of numerical and analytical calculations agree with the results of the conducted experiment. The expansion of effective sound insulation into frequencies higher than the eigen frequency of resonators has been confirmed.

3. The WI theory is essentially based on the method of homogenization - the replacement of discrete inhomogeneities in the waveguide wall with a continuous - "distributed" wall impedance. The experiment and numerical calculation showed that homogenization is applicable even with a small number of resonators, in this case three.

\section{References}

1. M . A . Issakovich, Theory of waveguide insulation of waves in long lines, All-U nion conference "W aves propagation and diffraction", Y erevan, 2 (1973) [in Russian]

2. A. D. Lapin, Soviet Phys. A coust. 21 (3), 337 (1975)

3. S.-H. Seo, Y .-H. K im, J. A coust. Soc. A m. 118 (4), 2332 (2005)

4. Z.G. Wang, S.H. Lee, C.K. Kim, C.M. Park, K. Nahm, S. Nikitov, J. A ppl. Phys. 103 (6) (2008)

5. C. Sugino, Y . X ia, S. L eadenham, M. Ruzzene, A. Erturk, J. Sound V ibr. 406, 104 (2017)

6. Guobiao Hua, Lihua Tang, X iaobin Cui, M ech. Syst. Signal Proces. 132, 595 (2019)

7. A. I. Komkin, A. I. Bykov, A coust. Phys. 62(3), 269 (2016)

8. M. Kalugin et al., Her. Bauman M osc. St. Tech. Univ. Ser. Natur. Scien. 3, 32 (2018)

9. A. E pikhin, M. K raposhin, K. V atutin The numerical simulation of compressible jet at low Reynolds number using OpenFOAM, E3S W eb of Conferences. EDP Sciences, 128, 10008 (2019)

10. S. M. Sivachev, L. L. M yagkov Thermomechanical Fatigue Analysis of Diesel Engine Piston: Finite Element Simulation and Lifetime Prediction Technique, in Proceedings of International Conference on Industrial Engineering. Springer, Cham, 109 (2019) 